\title{
Freezing of Triangulations
}

\author{
J.-P. Kownacki \\ Laboratoire de Physique Théorique et Modélisation, \\ CNRS-Université de Cergy-Pontoise, UMR 8089 \\ 5, mail Gay-Lussac, Neuville-sur-Oise, 95031 Cergy-Pontoise Cedex, France \\ E-mail : kownacki@ptm.u-cergy.fr
}

\begin{abstract}
Zero temperature dynamics of two dimensional triangulations of a torus with curvature energy is described. Numerical simulations strongly suggest that the model get frozen in metastable states, made of topological defects on flat surfaces, that group into clusters of same topological charge. It is conjectured that freezing is related to high temperature structure of baby universes.
\end{abstract}

Published in Eur. Phys. J B 38, 485-494 (2004) 


\section{Introduction}

Two dimensional dynamical triangulations models have been largely studied in the context of euclidean quantum gravity over the past decades (for reviews, see [1]). These models allow lattice regularization where the cut-off is the length of the links. In view of $2 \mathrm{~d}$ quantum gravity formulation, interesting features are essentially thermodynamical limits and critical static properties, as they are key features for continuum limit of lattice formulation. Great analytical progresses have been made but numerical simulations are particulary suited to these models and allow further investigations in remaining open problems. Square curvature terms in the action/hamiltonian is such a problem that still lacks an analytical solution.

However, as random surfaces models, dynamical triangulations can be used in several other domains like real membranes, foams, 2d-liquid, defects on surfaces, etc. [2] [3]. Dynamical triangulations models have the nice feature to be very simple to formulate - purely topological models - and to display very interesting physical behaviours.

Recently, Sherrington et al. have studied the dynamics of such a model with curvature energy. They discovered behaviours characteristic of strong glass formers [4]. They underlined the role of topological constraints in this model without quench disorder, and explained glassy behaviour as dynamics of topological defects at low temperature. Their aim was to study a simple model of supercooled liquid, but their results are not limited to it. In particular, dynamical properties are essential in numerical simulations, even if static properties are studied - as in 2D quantum gravity - as Monte-Carlo techniques consist in performing random walks in configuration space. In such a context, a glassy behaviour with exponentially long equilibration times can mask true equilibrium properties.

In this paper, the work described above is extended to zero temperature dynamics of a square curvature energy model. In fact, zero temperature limit, where no thermally activated process is allowed, is crucial to understand, as it gives more informations on the energy landscape and, thereby, on the whole low temperature dynamics.

\section{The model - Static properties}

\subsection{Definition}

The set of all regular two dimensional triangulations [5] with $N$ vertices, toroidal topology and no boundaries is considered. Regular means triangulations without tadpoles - vertex connected to itself - and self-energy diagrams - two vertices connected by two links. Moreover, each vertex is connected to at least three neighbours. This set is noted $T_{\mathrm{N}}^{\text {torus }}$. Topological constraints imply that the number of vertices $N$, the number of links $N_{l}$ and the number of triangles $N_{t}$ are not independent. The first constraint sets the Euler characteristic $\chi$ to zero for a torus. $\chi$ is a topological invariant depending on the number of classes of nontrivial loops on the surface - two for a torus. For a triangulation, it reads [6]:

$$
\chi=N-N_{l}+N_{t}
$$

so that, for a torus,

$$
N-N_{l}+N_{t}=0
$$


The second constraint is local: each triangle is made of three links and, if there are no boundaries, each link belongs to two triangles. So,

$$
2 N_{l}=3 N_{t}
$$

Combining relations (1) and (2) gives

$$
N_{l}=3 N \quad, \quad N_{t}=2 N
$$

In the model described here, each triangulation $\mathcal{T} \in T_{\mathrm{N}}^{\text {torus }}$ is endowed with one - and only one geometry. This is achieved by setting the length of all links to unity and imposing on metric to be flat inside each triangle of $\mathcal{T}$. Such geometries are called piecewise linear manifolds. It should be noticed that triangulations are not embedded in any larger space, so that geometries are internal.

A random surfaces model is built from this ensemble of surfaces with fixed topology but different geometries. The method consists to assign to each triangulation/geometry an energy $E(\mathcal{T})$ which governs dynamics and equilibrium. At equilibrium, each triangulation $\mathcal{T}$ is weighted by a Boltzman exponential $\exp (-\beta E(\mathcal{T}))$ with $\beta$ the inverse temperature. In the model, $E(\mathcal{T})$ depends on Gaussian - internal - curvature. As geometry inside each triangle is flat, curvature is concentrated at vertices. Following Regge calculus [7], an elementary surface $d s_{i}=q_{i}$ is defined at each vertex $(i)$ connected to $q_{i}$ neighbours (see figure 1 ). Such a vertex is called a $q_{i}$-vertex.

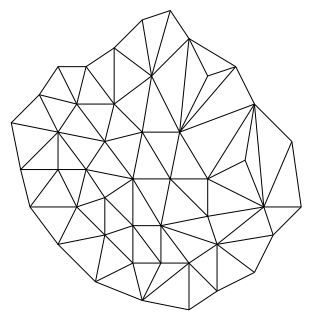

Figure 1: A triangulation.

Local curvature at vertex $(i)$ is $q_{i} R_{i}$, with

$$
R_{i}=\frac{\left(6-q_{i}\right)}{q_{i}}
$$

$R_{i}$ measures local deviation from flatness $\left(q_{i}=6\right.$ and $\left.R_{i}=0\right)$. However, $E(\mathcal{T})$ cannot be the total curvature $\sum_{i} q_{i} R_{i}$ because it is a topological invariant, as stated by the Gauss-Bonnet theorem, and would be a constant in the model. The choice for the energy is

$$
E(\mathcal{T})=\sum_{i}\left(q_{i}-6\right)^{2}
$$

It is not strictly speaking equal to the total square curvature

$$
\sum_{i} q_{i} R_{i}^{2}=\sum_{i} \frac{\left(q_{i}-6\right)^{2}}{q_{i}}
$$


but (3) and (4) are expected to give similar results, at least for small curvature, because

$$
\sum_{i} \frac{\left(q_{i}-6\right)^{2}}{q_{i}}=\frac{1}{6} \sum_{i}\left(q_{i}-6\right)^{2}-\frac{1}{36} \sum_{i}\left(q_{i}-6\right)^{3}+\ldots
$$

In both cases, surfaces are flattened by curvature energy at low temperature. However, expression (3) is more symetric with respect to $q_{i}=6$. Moreover, for surfaces containing only 5-, 6- and 7-vertices, the energy can be rewritten

$$
E(\mathcal{T})=n_{5}+n_{7}
$$

where $n_{k}$ is the number of $k$-vertices. As will be seen later, these surfaces play a crucial role at low temperature.

Finally, the partition function of the model reads

$$
Z_{N}(\beta)=\sum_{\mathcal{T} \in T_{N}^{\text {torus }}} \frac{1}{\mathrm{C}(\mathcal{T})} e^{-\beta E(\mathcal{T})}
$$

where $C(\mathcal{T})$ is a symetry factor needed to avoid overcounting symetric triangulations. For large $N$, $C(\mathcal{T})=1$ for almost all triangulations.

\subsection{Equilibrium Properties}

At infinite temperature $(\beta=0)$, the model has been solved [8] [9]. Typical surfaces are characterized by hierarchical structures of baby universes - bubbles growing on surfaces and linked by very small necks. The distribution of their size is known [10]. As a consequence, typical surfaces are fractal with Haussdorf dimension $d_{H}=4$ [11]. The distribution of vertices with $n$ neighbours $P(n)$ has been calculated [12] (see figure 2 ) and the variance is $\mu_{2}=10.5$, meaning a wide range of vertices on the surfaces. Note that the model follows a modified Aboav's law [13].

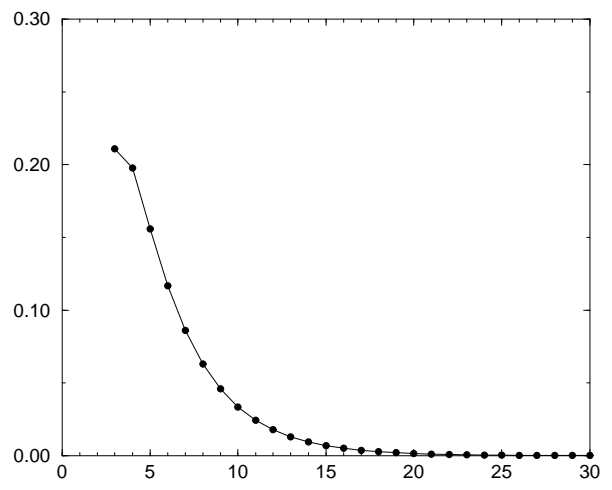

Figure 2: Equilibrium distribution $P(n)$ at $\beta=0$. 
At finite temperature, the model has not yet been solved and static properties have been studied numerically by many authors [14] [15]. However, a very similar model has been solved analytically, using matrix model techniques [16].

When temperature is lowered, curvature energy favours flattening of surfaces. The ground state of the model is the hexagonal lattice with $q_{i}=6$ at each vertex (figure 3 ). It should be emphasized that

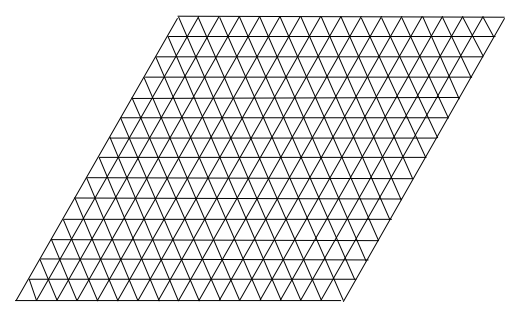

Figure 3: The hexagonal lattice.

the ground state does not depend on the precise form of the energy. In fact, $E(\mathcal{T})$ can be rewritten

$$
\begin{aligned}
E(\mathcal{T}) & =\sum_{i}\left(q_{i}{ }^{2}+36-12 q_{i}\right) \\
& =\sum_{i}\left(q_{i}{ }^{2}\right)+36 N-24 N_{l} \\
& =\sum_{i}\left(q_{i}{ }^{2}\right)-36 N
\end{aligned}
$$

so that, at given $N, E(\mathcal{T})$ is equivalent to $\sum_{i}\left(q_{i}-q_{o}\right)^{2}$, whatever the value of $q_{o}$. On the other hand, the mean neighbours number

$$
\begin{aligned}
<q_{i}> & =\frac{1}{N} \sum_{i} q_{i} \\
& =\frac{1}{N} 2 N_{l} \\
& =6
\end{aligned}
$$

is completely determined by topological constraints (1) and (2). So, the ground state is the configuration that minimizes $\sum_{i} q_{i}^{2}$ with $<q_{i}>=6$.

Here are summarized results obtained by numerical simulations, done as preliminary work in this paper, for sizes up to $N=65025$. Starting from hexagonal lattices, the system is initially equilibrated with typically 1000 sweeps, and simulations are carried with about 3000 sweeps. For details about the rules of simulations, see paragraph "dynamics" below. As can be seen in figure 4, the distribution $P(n)$ is more and more peaked at $n=6$ as temperature is lowered, meaning that surfaces contain essentially 6 -vertices. Then, 5- and 7-vertices can be seen as topological defects in flat surfaces, whose proportion vanishes as temperature is lowered. It should be noticed that, when only 5- , 6- and 7-vertices survive, $n_{5}=n_{7}$. It is a consequence of topological constraints (1) and (2), because

$$
N=n_{5}+n_{6}+n_{7}
$$




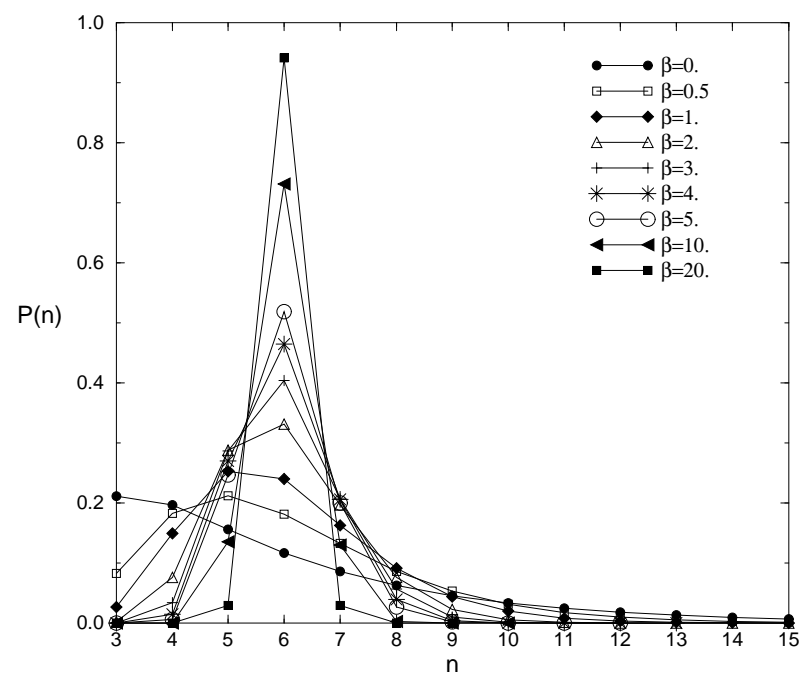

Figure 4: Equilibrium distribution $P(n)$ at various $\beta$, for $N=65025$.

$$
2 N_{l}=5 n_{5}+6 n_{6}+7 n_{7}
$$

so that

$$
5 n_{5}+6 n_{6}+7 n_{7}=6\left(n_{5}+n_{6}+n_{7}\right)
$$

hence

$$
n_{5}-n_{7}=0
$$

Baby universes also disappear at low temperature, as, otherwise, they would increase curvature energy. However, as can be seen in simulations, there is no sign of phase transition between high and low temperature phases. This is also suggested by dimensional analysis, as square curvature is not relevant in the infrared limit. Moreover, the same conclusion arises in the matrix model mentioned above.

\section{Dynamics}

Although static properties are rather simple, dynamics of the model reveals a very rich structure.

\subsection{Finite temperature}

\subsubsection{The rules}

The rules of dynamical evolution are based on the energy (3) and use so-called Alexander moves [17], more precisely $T_{1}$ moves, which consist in flipping a link in a triangulation (figure 5). Topology and 


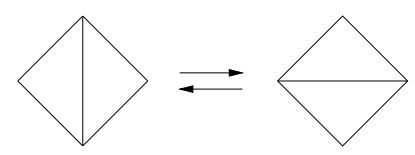

Figure 5: $T_{1}$ move.

number of vertices $N$ are conserved during evolution. It has been shown that $T_{1}$ moves allow ergodic explorations of the space of triangulations [12].

One elementary Monte-Carlo step of evolution is done as follows:

- Random choice of a link in triangulation $\mathcal{T}$;

- Flipping of the link with probability (Glauber type)

$$
w\left(\mathcal{T} \rightarrow \mathcal{T}^{\prime}\right)=\frac{1}{1+e^{\beta \Delta E}}
$$

where $\Delta E=E\left(\mathcal{T}^{\prime}\right)-E(\mathcal{T})$. The proposed triangulation $\mathcal{T}^{\prime}$ is rejected if it contains vertices with $q_{i}<3$, self-energy diagrams or tadpoles. At zero temperature $(\beta=+\infty)$, the transition probability becomes

$$
\begin{aligned}
& w\left(\mathcal{T} \rightarrow \mathcal{T}^{\prime}\right)=0 \quad \text { if } \Delta E>0 \\
& =\frac{1}{2} \text { if } \Delta E=0 \\
& =1 \text { if } \Delta E<0
\end{aligned}
$$

In the following, times are expressed in unit of sweeps, i.e $N_{l}$ successive elementary Monte-Carlo steps.

\subsubsection{Quench to low temperature}

Sherrington et al. [4] have studied the behaviour of the system after a quench from initial infinite temperature (disordered) state to low - but finite - temperature. Their main result is the emergence of glassy behaviour. It may looks rather surprising as the model presents neither frustration nor quenched disorder. There are two regimes in the evolution, depending on the temperature of the quench. For high temperatures, equilibrium is reached after a rapid relaxation, with equilibration times independent of the temperature.

On the other hand, for low temperatures, the system evolves in two steps. First, a rapid relaxation that corresponds to annihilation of all topological defects except 5- and 7-vertices, with fast decay of energy. Then, the system becomes glassy with equilibration times exponentially growing with inverse temperature (Arrhenius behaviour). In this second period, the energy reaches a plateau, while two times correlation functions of the curvature (local energy) develop shoulders and loose invariance in time translation. Glassy behaviour is the result of creations, annihilations and diffusions of 5- and 7-vertices. Simplified reaction-diffusion models give similar results [18]. The authors conclude that the model displays properties characterizing strong glass forming systems [19]. 


\section{Zero temperature and freezing}

The particular case of deep quench to zero temperature with infinite cooling rate [20] is now described. This is the original contribution of this paper.

\subsection{Energy}

The evolution of the energy $E(t)$ after a quench from infinite temperature state to zero temperature is plotted in figure 6 for several sizes of the system. Immediately after the quench, there is a very fast
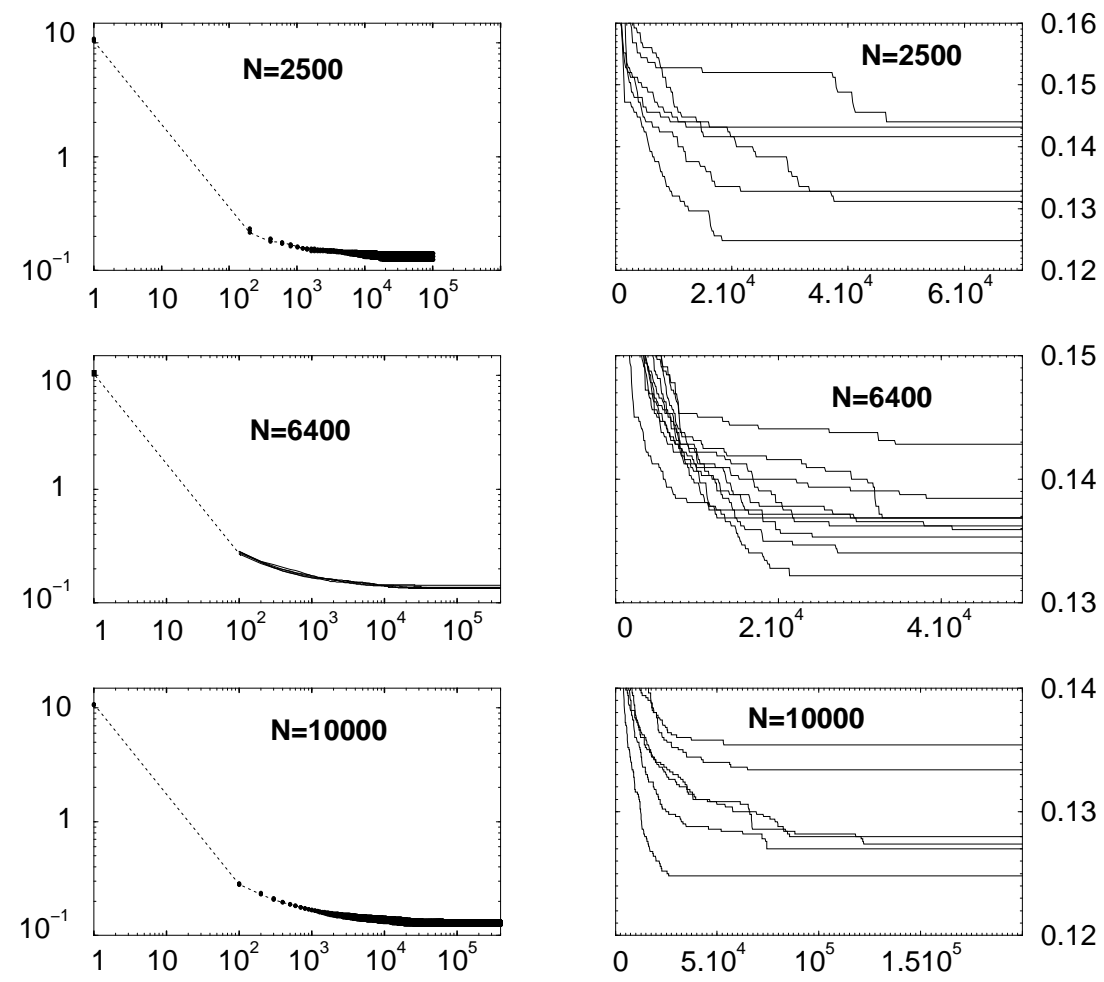

Figure 6: Evolution of energy after a quench to zero temperature, for $N=2500,6400$ and 10000. Each curve corresponds to one run.

decay of the energy, followed by a slowing down period where $E(t)$ reaches a serie of plateaux. At the beginning, this looks like stairs. But as time goes, the plateaux become broader and broader and eventually "infinite". It strongly depends on the size of the system. However, simple contemplation of the graph of $E(t)$ is not enough to know whether energy really stops or not. $E(t)$ could as well decrease to lower plateaux, and eventually to zero, after very long times unreachable by reasonable computer simulations. However, as will be shown in the following, it is very likely that, after a deep quench, the system gets trapped in metastable states. 
The final value reached by the energy follows a sharp distribution centered on a mean value $E_{f}$, according to initial disordered states. For each data point in figure 7, 16 independent runs are performed with 1000 heating sweeps at infinite temperature followed by sufficient sweeps at zero temperature (up to $5.10^{5}$ sweeps for $N=12500$ ) - depending on the time needed by energy to get frozen. The first important point is that $E_{f}$ is strictly greater than zero: the system is trapped in metastable states and never goes to its ground state. In other words, the energy freezes, in a time that depends on the size of the system. This can be seen by looking at the evolution of $P(n)$, which shows a very fast invasion of surfaces by 6 -vertices. Both descriptions - in terms of energy and in terms of defects - are equivalent as energy and number of defects $n_{\text {defects }}$ are simply related, if defects are 5and 7-vertices only:

$$
E=n_{\text {defects }}
$$

The second important point is that $E_{f} / N$ is constant for large $N$, as can be seen in figure 7. A power law fit gives a limiting value $\lim _{N \rightarrow \infty} E_{f} / N=0.1342(4)$. So, mean frozen energy per site is a thermodynamical characteristic of the model. Alternatively, a finite fraction - 13\% - of defects remains in frozen states.

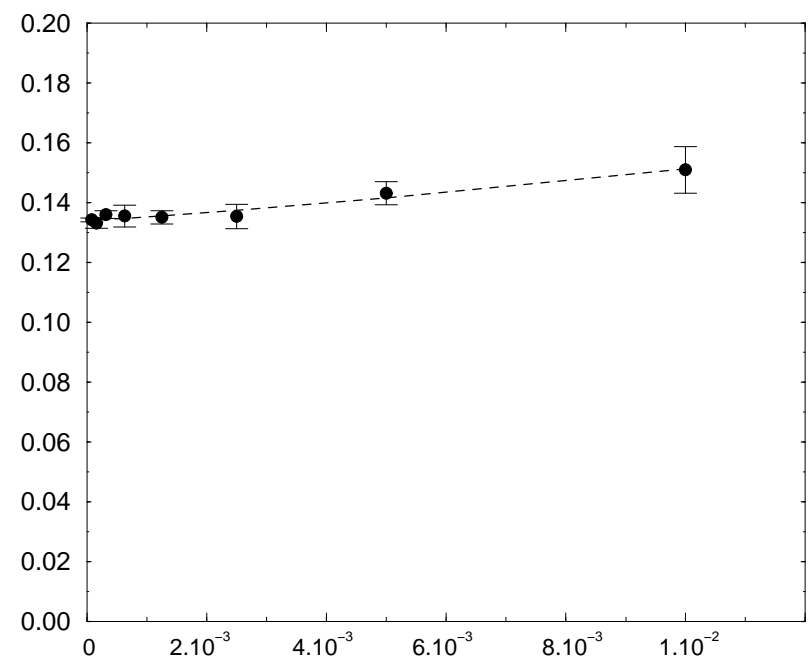

Figure 7: Frozen energy per site $E_{f} / N$, versus $\frac{1}{N}$. The dashed line indicates a power law fit.

\section{2 $T_{1}$ Moves}

At zero temperature, besides 6-vertices, only 5- and 7-vertices survive. Evolution of the system can be seen as dynamics of such defects through the six possible $T_{1}$ moves shown in figure 8 . Moves $d$ ), $e$ ) and $f$ ) have negative energy balance $(\Delta E<0)$ and correspond to annihilation of defects. The others $-a), b)$ and $c$ ) - can be viewed as diffusion of defects on the surface. In this second category, moves $a$ ) and $b$ ) - called flippers in the following - perform local rotations of 5-5 or 7-7 pairs. On the other hand, move $c$ ) is a true diffusion of a dipole - i.e a pair of 5-7. In order to understand the evolution of 

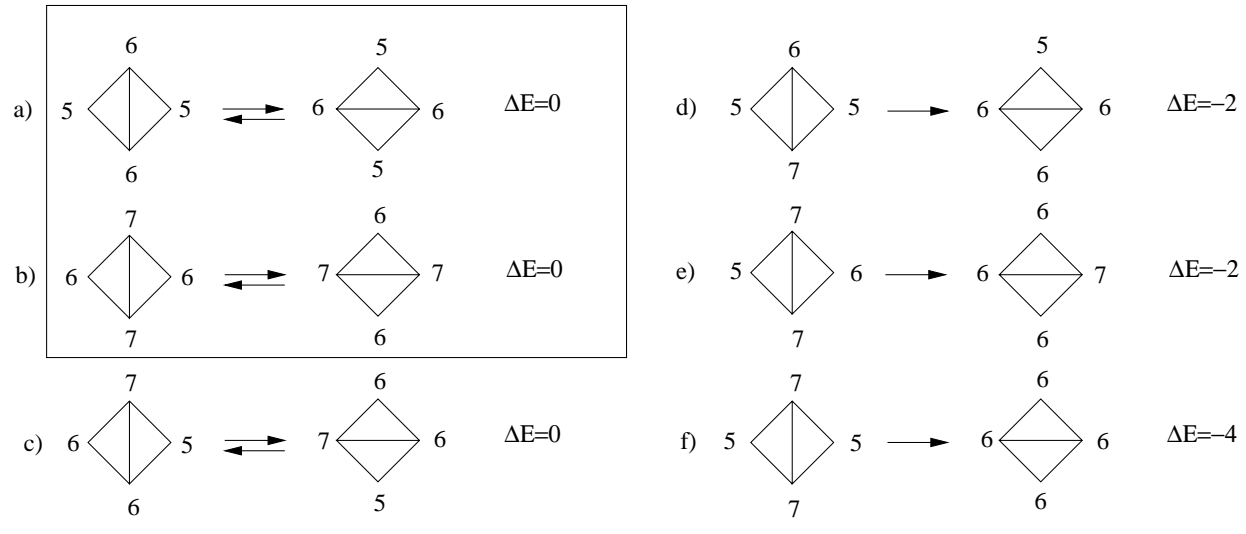

Figure 8: $T_{1}$ moves allowed at zero temperature. When there are no dipoles (5-7), only the two first moves (in the box) can occur.

the system after a deep quench, it is important to know the possible ways of instantaneous evolution of the system at a given time. This depends on the repartition of $T_{1}$ moves and gives a partial view on the energy landscape [21]. More precisely, at a given time $t$, it is possible to group the links of the surface into three categories: the first one $(I)$ contains links that cannot be moved because energy would be increased; the second one $(I I)$ contains links whose moves make energy strictly lower if they are flipped; and the third one $(I I I)$ contains links that let energy unchanged. Two subcategories of $(I I I)$ can be defined: (IIIa) contains links in $(I I I)$ correponding to dipole diffusion, and $(I I I b)$ contains links in $(I I I)$ corresponding to flippers. Proportions of links in $(I),(I I),(I I I a)$ and $(I I I b)$ at time $t$ after a deep quench are respectively written $w_{I}(t), w_{I I}(t), w_{I I I a}(t)$ and $w_{I I I b}(t)$. They are plotted for different sizes in figure 9 , obtained by averaging about 8 independent runs for each size. It is not surprising to see that $w_{I}(t)$ grows rapidly and, conversely, that $w_{I I}(t)$ decreases rapidly: this is simply the evolution toward the ground state - where all links are in $(I)$ - as expected for a system at zero temperature. However, after this first rapid evolution - corresponding to rapid decay of energy there is a slowing down period where $w_{I I}(t)$ is very small, meaning that the system can hardly lower its energy. The reason is that the system is almost unable to found local configurations that could lead to $d), e$ ) or $f$ ) moves. On the other hand, $w_{I I I a}(t)$ is strictly greater than zero, so that diffusions of dipoles still occur. Through these diffusions, dipoles can eventually get close to other defects and give local configurations of type $(I I)$. However, creation of such configurations by this mechanism is rather unlikely because dipoles are rare in this phase (see figure 10). As a consequence, the dynamics is very slow. This mechanism is confirmed by carefully looking at $w_{I I}(t)$ and $w_{I I I a}(t)$ simultaneously (see figure 11). Small peaks of $w_{I I}(t)$ occur immediately after small peaks of $w_{I I I a}(t)$, meaning that diffusion of pairs is the only way to create local configurations leading to energy decrease. So, the role of $(I I I a)$ is crucial in the slowing down period.

However, after some time, $w_{I I}$ and $w_{I I I a}$ become equal to zero: this coincides with energy freezing and removal of dipoles. But the dynamics is not really stopped, because during the slowing down period described above, $w_{I I I b}$ reaches a plateau with small but finite value - independant of the size - 

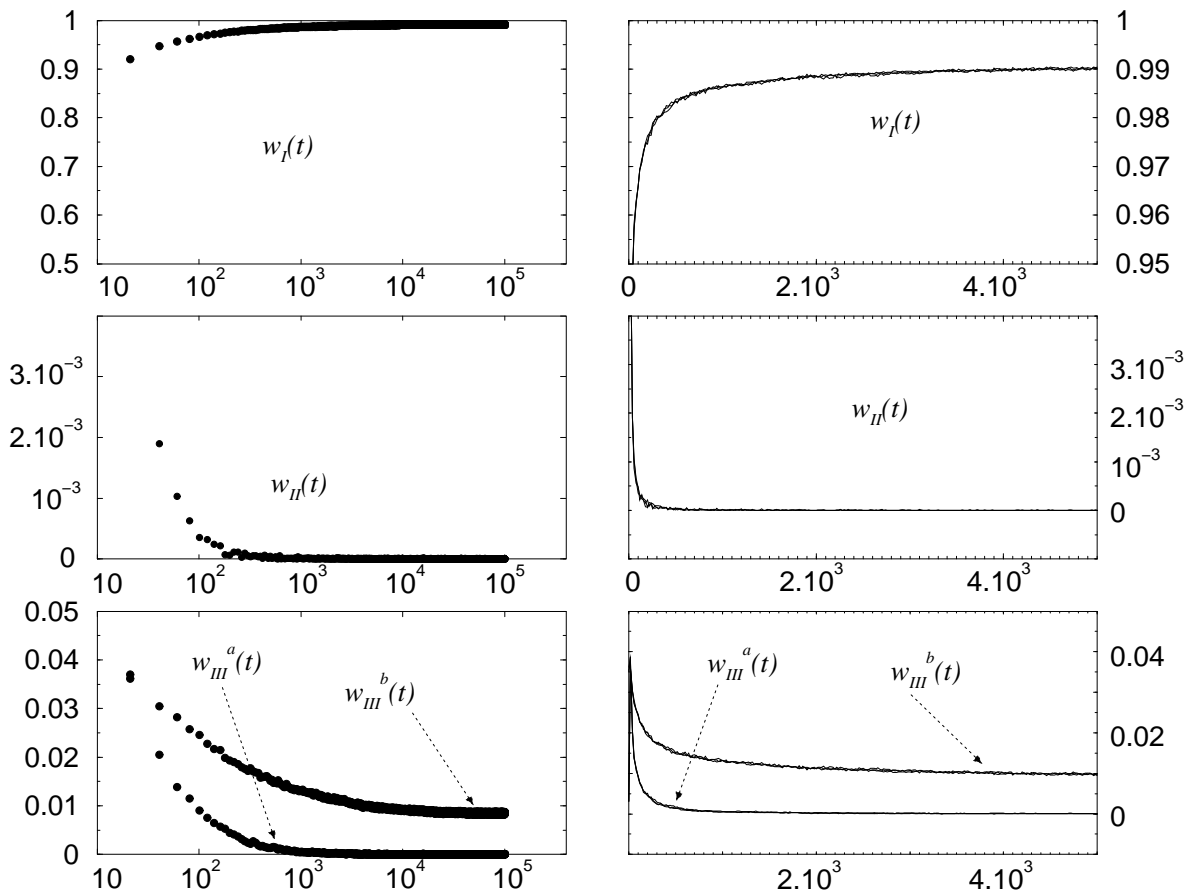

Figure 9: Probabilities of flips $w_{I}, w_{I I}, w_{I I I}^{a}$ and $w_{I I I}^{b}$ versus time for $N=2500,4900$ and 10000. Curves corresponding to different sizes are not distinguishable.

so that flippers still occur even after removal of dipoles and energy freezing.

At this point, the problem is to know whether flippers eventually allow diffusion of defects, through some collective moves, that could lead, after a long time, to creation of dipoles. In this case, energy could decrease again, and freezing of energy would be an illusion due to short time measurements.

\subsection{Geometric description}

In order to elucidate the role of flippers, it is necessary to have spatial informations on surfaces when removal of dipoles and energy freezing - at least apparently - occur. So, it is very instructive to draw the surfaces. As can be seen in figure 12, not only do the defects of different kind - i.e 5- and 7-vertices - separate, but, conversely, defects of the same kind group together into clusters. More precisely, frozen surfaces look like a sea of 6 -vertices with isolated clusters of defects containing either 5 -vertices or 7-vertices. Figures 13 and 14 show the size distributions of these clusters, measured on 16 independent frozen surfaces obtained after 1000 sweeps of thermalization followed by sufficient sweeps (up to $4.10^{5}$ ) at zero temperature. It should be noticed that the sizes do not exceed few vertices. Moreover, the distribtution itself does not depend on the size of the surfaces, meaning that the mechanism of freezing is local and not influenced by long range correlations. 


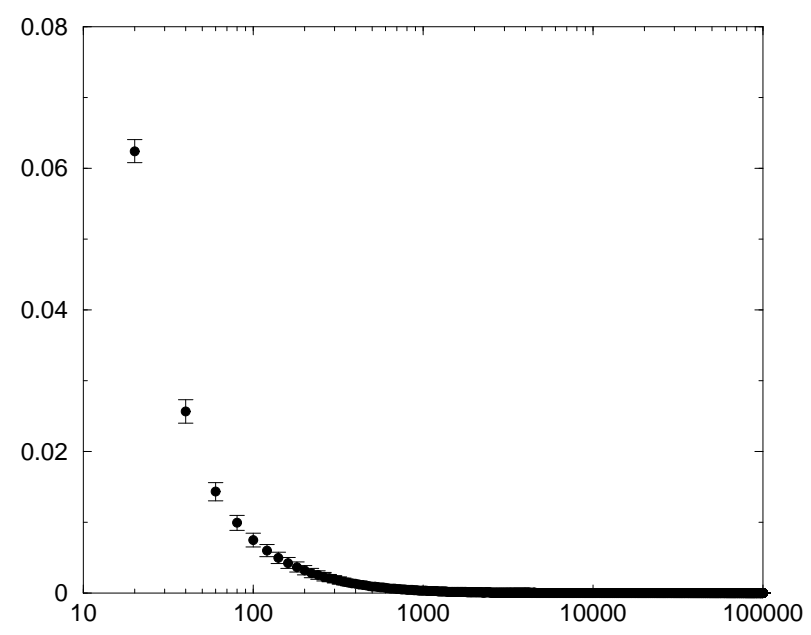

Figure 10: Fraction of dipoles versus time for $N=2500$, averaged on 576 independent runs.

\subsection{Flippers but no dipole creation}

The issue is now to understand the role of flippers on a surface made of 5- and 7-clusters inside a sea of 6 -vertices. There are two possibilities:

- 5-vertices and 7-vertices diffuse across the whole surface and, eventually, some of them are close enough to form dipoles and allow decreasing of energy. In this case, evolution could be very slow, but the system is not really frozen;

- 5-vertices and 7-vertices are moving but clusters are confined within spatial boundaries so that no dipoles are created. In this case, the system is for ever trapped in a set of metastable states.

Arguments suggesting that flippers cannot lead to dipole creation are now given. The starting point is a state with clusters of different kinds (5-clusters and 7-clusters), inside a sea of 6 -vertices (about $85 \%$ of the surface).

The 5-clusters have the following property: if there exists a convex contour $\mathcal{C}$ surrounding a 5cluster and containing no 7-vertex, then, at zero temperature, $\mathcal{C}$ will never be crossed by a 5-vertex coming from the cluster, i.e there will never be 5-vertices outside $\mathcal{C}$. Convex contour means that the number of links attached to each vertex of $\mathcal{C}$ and going outside of $\mathcal{C}$ is larger or equal to the number of links going inside $\mathcal{C}$ - the links of $\mathcal{C}$ are not taken into account. The proof runs as follows :

Let a region $\mathcal{R}$, containing only 5 - and 6 -vertices, be bounded by a loop $\mathcal{C}$, made of links connecting 5 - and/or 6 -vertices. It is supposed that there are only 6 -vertices outside $\mathcal{C}$. Then, moves of type $a$ ) are performed in $\mathcal{R}$ :

- The boundary $\mathcal{C}$ cannot be broken: consider a link $l \in \mathcal{C}$. The only possibility for $l$ to be moved is to belong to type $(I I I b)$ (flipper). In this case, because of the convexity of the contour, one of the two neighbouring 5 -vertices is inevitably outside $\mathcal{C}$. It would contradict the starting hypothesis: so, $l$ cannot be moved.

- Consider now a set of four vertices $\left\{\alpha_{1}, \beta_{1}, \alpha_{2}, \beta_{2}\right\}$ involved in a move of type $a$ ), where $\alpha_{1}, \alpha_{2}$ are 5 -vertices and $\beta_{1}, \beta_{2}$ are 6 -vertices. As shown above, the link $\beta_{1}-\beta_{2}$ cannot belong to $\mathcal{C}$. After the $T_{1}$ 

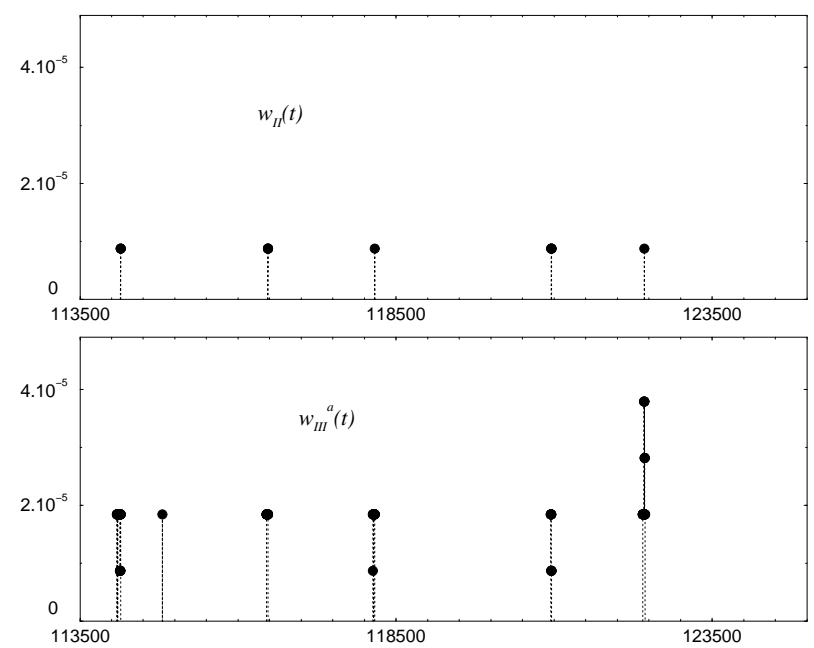

Figure 11: Detail of $w_{I I}$ and $w_{I I I}^{a}$ for one run and $\mathrm{N}=4900$.

move, $\alpha_{1}, \alpha_{2}$ are 6 -vertices and $\beta_{1}, \beta_{2}$ are 5-vertices. There are two possibilities that could lead to a diffusion of 5 -vertices outside $\mathcal{C}$ :

(i) $\beta_{1}$ and $\beta_{2}$ are outside $\mathcal{C}$ or

(ii) $\beta_{1}$ or $\beta_{2}$ are outside $\mathcal{C}$.

Of course, the starting hypothesis prevents $\alpha_{1}$ and $\alpha_{2}$ to be outside $\mathcal{C}$. So, for the case $(i), \mathcal{C}$ must contain $\alpha_{1}$ and $\alpha_{2}$, but neither $\beta_{1}$ nor $\beta_{2}$. It is easy to see that such a configuration implies a non convex boundary $\mathcal{C}$. For the case $(i i), \mathcal{C}$ must contain $\alpha_{1}, \alpha_{2}$ and $\beta_{1}$ (resp. $\beta_{2}$ ) but not $\beta_{2}$ (resp. $\beta_{1}$ ). It also implies a non convex boundary at $\beta_{1}$ (resp. $\beta_{2}$ ).

To conclude, diffusion of 5 -vertices outside $\mathcal{C}$ is not allowed.

Consider now the 7-clusters. Unfortunately, last arguments given for 5-clusters do not work. In particular, a convex boundary can be deformed by a flipper. However, it is possible to have a rather reliable result. Consider a 7 -cluster and perform - by computer simulations - $T_{1}$ moves of type $b$ ) only in this cluster. Then, the pattern of the cluster evolves through flippers but, as can be seen by drawing surfaces, it does not extend much and never reaches any 5-cluster. These simulations take very few computer time because 7-clusters contain only fews vertices. So, it is possible to perform very extensive runs and to have great confidence level in this result.

To summarize, it is very likely that the system gets trapped and follows a constant energy walk in a set of states; these states are made of 5- and 7-clusters and are subject only to flippers that do not destroy the cluster structure - in particular, flippers cannot create dipoles.

\section{Inherent structures and energy landscape}

The quenching procedure to zero temperature used in this work maps equilibrium states to frozen sets of metastable states. It is in some sense a steepest-descent dynamics to inherent states as defined by Stillinger and Weber [22]. This approach of glassy behaviours is based on a decomposition of the 


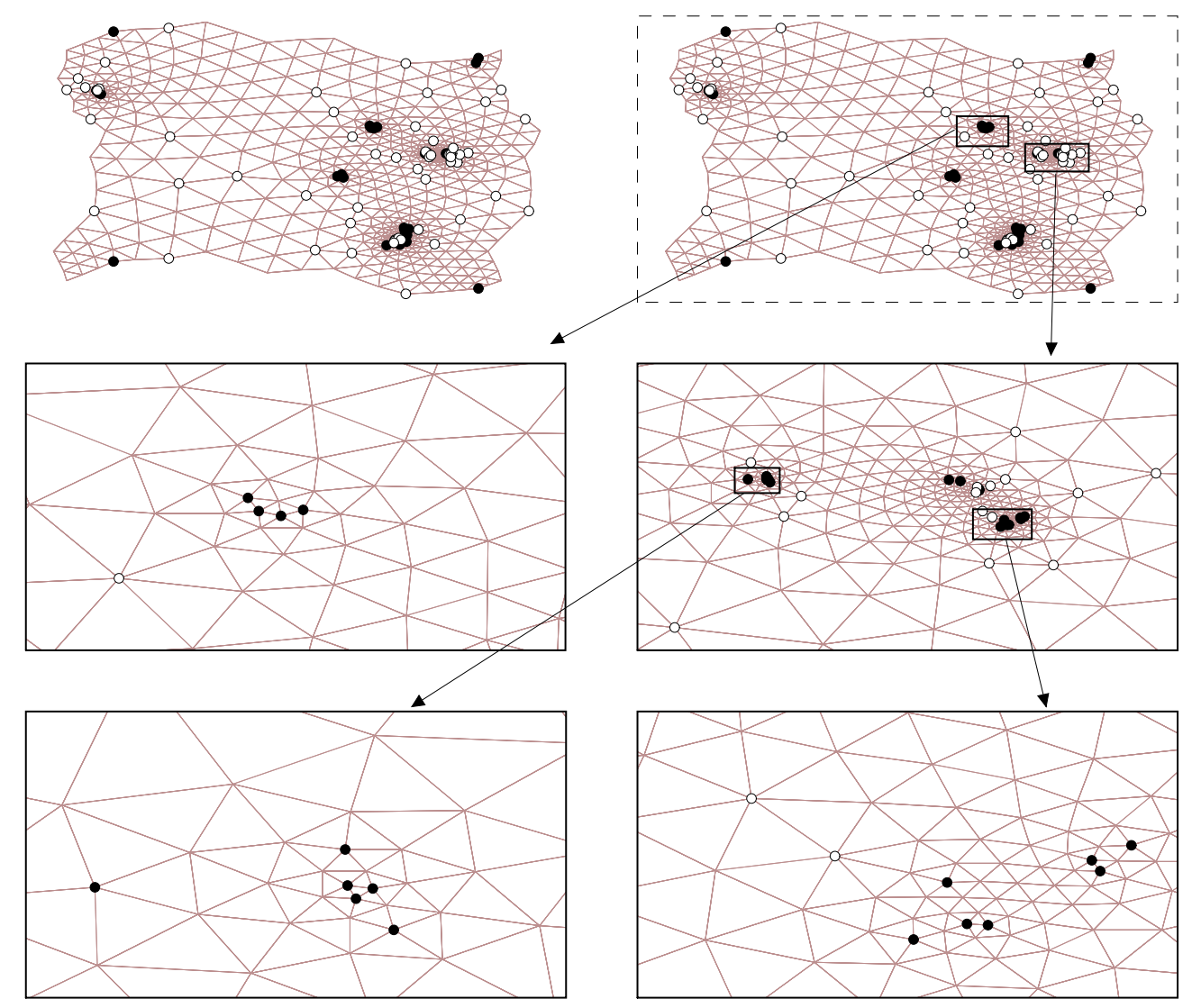

Figure 12: A frozen surface with $N=900$. 5-vertices are represented by $\bullet$ and 7 -vertices by $\circ$. All others vertices are 6 -vertices. Arrows indicate zooms of regions in the boxes.

configuration space into basins, called inherent structures. Each basin contains the states mapped through steepest-descent to the same local minimum in the energy landscape. This method provides a deeper understanding of glassy behaviours in terms of fast intra-basins and slow inter-basins dynamics, with the definition of a configurational entropy counting the number of inherent structures with a given energy or, alternatively, a given free energy [23]. However, this symbolic dynamics between inherent structures is not necessarily relevant for of all glassy systems [24] [25].

For the model studied in this paper, it would be very interesting to use the Stillinger and Weber approach. But, as the model is discrete, the zero temperature dynamics is not a deterministic mapping to metastable states. Moreover, these inherent structures are degenerate, as shown in the previous section. So, it would require slight modifications to define unambiguously concepts like inherent structures, configurational entropy ... The present work is a first step in this approach as it provides a characterization of the metastable states reached after a quench from infinite temperature equilibrium states. To go further, the frozen energy per site following a quench from finite temperature to zero temperature has been calculated for rather small surfaces $(N=400)$, as preliminary result. Figure 


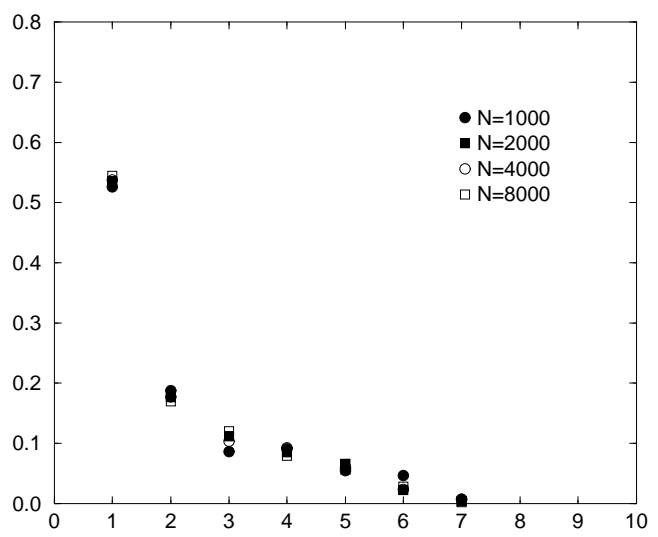

Figure 13: Size distribution of 5-clusters on frozen surfaces.

15 shows the variation of $E_{f}(T) / N$ with temperature. As can be seen, this quantity is more or less independent of $T$ for $T \geq T_{o}$ with $T_{o} \simeq 2$, and strongly decreases for $T<T_{o}$, meaning that the system explores deeper valleys in the energy landscape.

\section{Conclusion}

The model of random surfaces with elastic curvature energy studied in this paper shows rather trivial static properties. In particular, there is no phase transition driven by curvature coupling constant curvature energy simply smoothes surfaces and the whole fractal structure of baby universes disappears at low temperature equilibrium.

However, the system reveals complex dynamical behaviour, though there is no frustration nor quenched disorder. In particular, when the system is quenched from totally disordered infinite temperature state to low temperature, glassy behaviour takes place and relaxation time becomes exponentially long as temperature is lowered.

Glassy behaviour is dramatically strengthened for deep quench at zero temperature. In this case, simulations and some arguments strongly suggest that the system really freezes: after a rapid decay, the energy stops and the ground state is never reached. Equivalently, freezing corresponds to defects (5- and 7-vertices) that cannot be removed by the system. This property does not depend on the size of the system and should be still valid at the thermodynamical limit. In fact, even if energy stops, there is a remaining dynamics of the system, consisting in flippers, i.e $T_{1}$ moves that locally rotates defects of same kind. But flippers cannot make energy evolve because of the spatial repartion of defects: they group together into clusters made of one kind of defects . Then, flippers cannot lead to diffusion of defects outside some bounded regions, and dipoles, that could lower energy, are not created. The system is forever trapped in a set of metastable states. Similar results are obtained for reaction-diffusion models [18]. However, there is still an open question: why do defects of same kind group together into clusters? In particular, it would be very interesting to study the evolution of the fractal structure of baby universes during the deep quench. At infinite temperature, it 


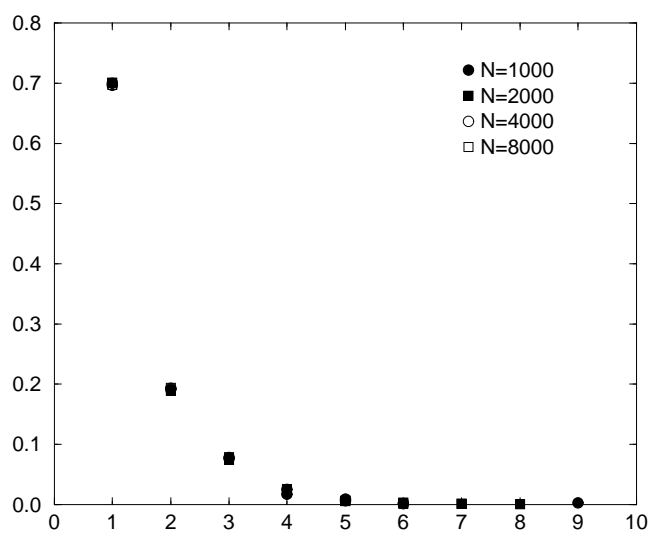

Figure 14: Size distribution of 7-clusters on frozen surfaces.

is known that $d_{H}=4$. In frozen states, there are no more baby universes. But preliminary results suggest that the fractal dimension of frozen surfaces is significantly greater than 2 - the value for flat surfaces. So, a guess can be made: frozen surfaces keep memory of their initial baby universes structure through structure of clusters. The mechanism of clusters creation would be the following : each initial baby universe is more or less isolated from the rest of the surface; then, just after a quench to zero temperature, each baby universe tries to reach its own local ground state. But in each baby universe, the difference between the number of 5 -vertices and the number of 7 -vertices is topologically constrained by the boundary of the baby universe. So, after a rapid multi-relaxation period where boundaries of baby universes are not expected to be much modified, some regions would be left with an excess of 5 -vertices and some others with an excess of 7 -vertices. This mechanism could lead to creation of clusters. To summarize, clusters structure would be a trace of initial baby universes structure.

It would be very interesting to use the inherent structures approach of Stillinger and Weber for the system studied here, and to see wether this method can provide a better understanding of the glassy behaviour observed at finite temperature. The present work is in some sense a first step in this direction.

\section{Acknowledgements}

I am grateful to C. Godrèche for his help, suggestions and encouragments all along this work. I would like to thank J-M Luck and J-M Drouffe for interesting discussions and suggestions. I wish to acknowledge F. David for helpfull discussions about strong coupling expansion of planar graphs. Finally, I would like to thank the laboratory SPEC at CEA Saclay where part of this work was done using computer facilities. 


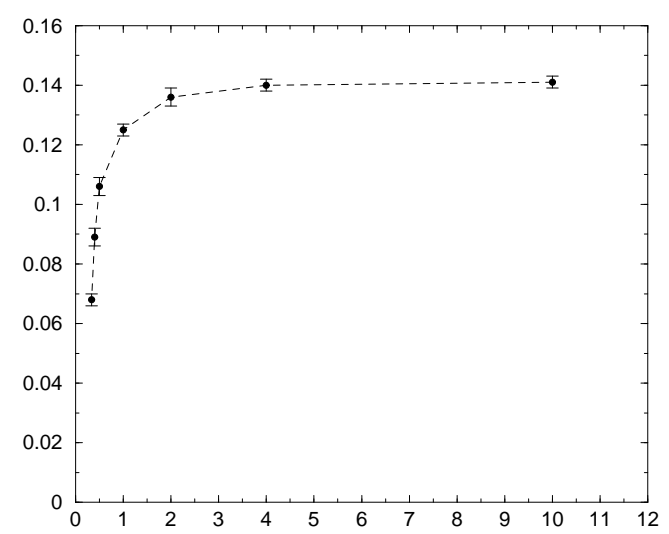

Figure 15: Frozen energy per site $E_{f} / N$ versus temperature, for $N=400$.

\section{References}

[1] F. David, in Gravitation and Quantizations, (Les Houches Summer School, Session LVII, 1992) 679-750, edited by B. Julia and J. Zinn-Justin (Elsevier, Amsterdam, 1995);

J. Ambjørn, in Fluctuating Geometries and Field Theory, (Les Houches Summer School, Session LXII, 1994) 77-193, edited by F. David, P. Ginsparg, and J. Zinn-Justin (Elsevier, Amsterdam, 1996) ;

P. Di Francesco, P. Ginsparg and J. Zinn-Justin, Phys. Rep. 254 (1995), 1;

J. Ambjørn, B. Durhuus, and T. Jónsson, Quantum Geometry, Cambridge University Press, (1997).

[2] Statistical Mechanics of Membranes and Surfaces, Proc. of the Fifth Jerusalem Winter School for Theoretical Physics, 1987/1988, eds. D. Nelson, T. Piran, and S. Weinberg, World Scientific, Singapore (1989).

[3] M.J. Bowick, S.M. Catterall and G. Thorleifsson, Phys.Lett. B391 (1997), 305.

[4] T. Aste and D. Sherrington, J. Phys. A32 (1999), 7049;

L. Davison and D. Sherrington, J. Phys. A33 (2000), 8615;

L. Davison, D. Sherrington, J. P. Garrahan and A. Buhot, J. Phys. A34 (2001), 5147;

A. Buhot, J.P. Garrahan and D. Sherrington, J. Phys. A36 (2003), 307.

[5] J. Frölich, in Lectures Notes in Physics, Vol. 216, ed. L. Garrido, Springer (1985);

F. David, Nucl. Phys. B257 (1985), 45;

V.A. Kazakov, Phys. Lett. B150 (1985), 182.

[6] C. Itzykson and J-M. Drouffe, Statistical Field Theory, Vol. 2, Cambridge University Press, (1990).

[7] T. Regge, Nuovo Cimento 19 (1961), 558.

[8] W.T. Tutte, Can. J. Math. 14 (1962), 21.

[9] E. Brézin, C. Itzykson, G. Parisi and J.B. Zuber, Comm. Math. Phys. 59 (1978), 35.

[10] S. Jain and S.D. Mathur, Phys. Lett. B286 (1992), 239. 
[11] H. Kawai, N. Kawamoto, T. Mogami and Y. Watabiki, Phys. Lett. B306 (1993), 19.

[12] D.V. Boulatov, V.A. Kazakov, I. K. Kostov and A.A. Migdal, Nucl. Phys. B275 (1986), 641.

[13] C. Godrèche, I. Kostov and I. Yekutieli, Phys. Rev. Lett. 69 (1992), 2674.

[14] D.V. Boulatov and V.A. Kazakov, Phys. Lett. B184 (1987), 247.

[15] N. Tsuda and T. Yukawa, Phys. Lett. B305 (1993), 223;

T. Yukawa, N. Tsuda and A. T. Sornborger, Nucl. Phys. B(Proc. Suppl.)30 (1993), 791;

S. Ichinose, N. Tsuda and T. Yukawa, Nucl. Phys. B445 (1995), 295;

S. Ichinose, N. Tsuda and T. Yukawa, Int. J. Mod. Phys. A12 (1997), 757.

[16] V.A. Kazakov, M. Staudacher and T. Wynter, Nucl.Phys. B471 (1996), 309.

[17] J.W. Alexander, Ann. Math. 31 (1930), 292;

M. Gross and S. Varsted, Nucl. Phys. B378 (1992), 367.

[18] J-M. Drouffe, private communication.

[19] W. Kob, J. Phys. Condens. Matter 11 (1999), R85.

[20] S. Sastry, P. G. Debenedetti and F. H. Stillinger, Nature 393 (1998), 554.

[21] K. Broderix, K. K. Bhattacharya, A. Cavagna, A. Zippelius and I. Giardina, Phys. Rev. Lett. 85 (2000), 5360.

[22] F.H. Stillinger and T.A. Weber, Phys. Rev. A25 (1982), 978.

[23] F. Ritort and P. Sollich, Advances in Physics 52 (2003), 219;

B. Coluzzi, A. Crisanti, E. Marinari, F. Ritort and A. Rocco, Eur. Phys. J. B32 (2003), 495;

A. Crisanti and F. Ritort, J. Phys.: Condens. Matter 14 (2002), 1381.

[24] A. Crisanti, F. Ritort, A. Rocco and M. Sellitto, J. Chem. Phys. 113 (2000), 10615;

A. Crisanti, F. Ritort, A. Rocco and M. Sellitto, J. Phys.: Condens. Matter 14 (2002), 1523.

[25] G. Biroli and J. Kurchan, Phys. Rev. E64 (2001), 016101. 